\title{
Culturally Effective Practice With Refugees in Community Health Centers: An Exploratory Study
}

\author{
Nicole Dubus \\ Ashley Davis
}

\begin{abstract}
The global refugee crisis requires providers of health and behavioral health services to develop culturally-effective practices that can meet the needs of the everchanging demographics of those being resettled. Community health centers in the United States are often asked to provide services during the first year of resettlement for refugees. Social workers are among those professionals who provide the behavioral health services in the community health centers. To better understand the challenges for these providers, this qualitative study examines the experiences of 15 providers of refugee behavioral health services at community health centers in the northeast of the United States. The participants were interviewed, and those transcribed interviews were analyzed for themes. Findings revealed three main themes: client engagement as crucial; collaboration with interpreters; and cultural competence is an imperative but ill-defined. Important implications focus on the need for cultural competence and the challenge to obtain this competence given the resources and demands in community health centers.
\end{abstract}

Keywords: Refugee; community health center; cultural competence

Currently, there are over 65 million forced migrants throughout the world (United Nations High Commissioner for Refugees [UNHCR], 2017). Forced migrants are individuals that are required to relocate due to internal conflicts, persecution, or unsustainable environments due to social/economic factors or climate changes. Of the 65 million forced migrants, over 23 million are United Nations designated refugees. The current refugee crisis is the largest in history and is not abating. In fact, by all statistics it is increasing and rapidly changing (UNHCR, 2017). With the effects of climate changes accelerating, one can expect forced migrations due to climate changes to increase (Campbell, 2014; Farbotko, Stratford, \& Lazrus, 2016; Yoosun, 2008), adding to our current refugee crisis.

While the legal term "refugee" has been in use since 1951, the United States, and many other countries, have been receiving refugees for centuries (Matera, Stefanile, \& Brown, 2015). Throughout much of the history of the United States, social workers have in some form or other been providing direct services to refugees (Healy, 2008). Our long history of working with refugees continues today. Each year the president determines the number of refugees allowed to enter the United States (UNHCR, 2017). In 2016, under President Obama, the United States, accepted nearly 85,000 refugees (Krogstad \& Radford, 2017). In 2017, President Trump set the cap to 45,000 refugees, and in 2018, the cap was placed at 21,292 refugees to be accepted into the United States (UNHCR, 2017). While at the time of this writing, the numbers of new refugee arrivals have been restricted, the global crisis has not abated, and the needs of refugees will continue to be a dynamic and demanding

Nicole Dubus, PhD, MSW Assistant Professor, Social Work. San Jose State University, San Jose CA, 95112. Ashley Davis, Associate Professor, Social Work, Boston University, Boston MA.

Copyright @ 2018 Authors, Vol. 18 No. 3 (Spring 2018), 874-886, DOI: 10.18060/21731 (cc) BY This work is licensed under a Creative Commons Attribution 4.0 International License. 
concern for social workers well beyond any current presidential administration.

In the United States, community health centers are on the front lines of working with refugees. A community health center is a nonprofit comprehensive primary care facility that is federally mandated to provide services to underserved areas (Taylor, 2004). Through the assistance of local agencies, the UN-designated refugee is connected to a local community health center that provides health and behavioral health care services (Bruno, 2015; Morland \& Levine, 2016). Refugees have often survived traumatic events, leaving many with symptoms of depression, anxiety, and post-traumatic stress disorder. All refugees need housing, support entering into the labor field, and many need assistance learning English (Esses, Hamilton, \& Gaucher, 2017; Moulton, 2016). In addition, individuals and families may enter the United States with pre-existing health and mental health conditions that can complicate their resettlement (Burnett, 2016; Bustamante, Leclerc, Mari, \& Brietzke, 2016; Ellis, Murray, \& Barrett, 2014; Hansen \& Huston, 2016; Moulton, 2016). Social workers are among the healthcare professionals who serve refugees in community health centers and must help them navigate an under-resourced system to meet their needs (Adams, Gardiner, \& Assefi, 2004; Al-Husban \& Adams, 2016; Ay, Arcos González, \& Castro Delgado, 2016; Barghadouch et al., 2016; Bozorgmehr, Szecsenyi, Stock, \& Razum, 2016; Burnett, 2016; Esses et al., 2017).

\section{Challenges in Cross-Cultural Social Work Interventions}

This study examines in particular the cultural challenges of providing effective interventions. In this section we introduce some of the major cultural challenges that other studies have researched. Refugees may come from situations and cultures not accustomed to seeking mental health services. Providing culturally-effective services to this group may also involve addressing possible stigma the individual, family or community may experience when seeking mental health services (Al-Makhamreh, Spaneas \& Neocleous, 2012). Additionally, some cultures may be more family-centric than the United States, and therefore may expect to involve family members in their care (Weaver, 2005; Weine, Kuc, Dzudza, Razzano, \& Pavkovic, 2001). For social workers, this can create ethical issues regarding privacy and confidentiality. Service delivery may need to be modified to be culturally-effective. Weaver (2005) posits that group-based interventions grounded in cultural competency and spirituality could more effectively provide support to refugees. In a meta-analysis of refugee programs, Sossou, Craig, Ogren, and Schnak (2008) found that some recipients of refugee services were more responsive to a "contact perspective approach", a cross-cultural approach that involves an exchange of ideas, negotiation, and interaction among refugees, their families, and professionals.

UN designated refugees are provided Medicaid to cover health expenses their first year of resettlement. However, many are prevented from receiving care because of communication and transportation barriers (Fike \& Androff, 2016). Social workers are crucial players in knitting together services and supporting refugee communities. In addition to collaboration, outreach, and culturally-sensitive practice, work with refugees requires language services and experience in working with interpreters as cultural brokers (Decola, 2011; Fondacaro \& Harder, 2014; Hsieh \& Kramer, 2012; Njeru et al., 2016). The issues involved in working with refugees are complex. Social workers in community health 
centers are on the front lines of managing the needs of newly arrived refugees. It is important to understand more about these challenges. Our current refugee crisis provides a painful and critical moment to learn best-practices, and to share those practices with others in the field. This study is timely and important in expanding the field's knowledge on the best practices in working with refugees in community health centers.

\section{Purpose of the Study}

This study sought to understand how community health centers are responsive to differing refugee populations. Understanding the experiences of community health center social workers working with refugees, may provide helpful information on best practices and current challenges. This article reports on a descriptive study of how social workers in community health centers perceive their provision of services to refugees across cultural and linguistic barriers. This study examines community health centers $(n=15)$ in six northeastern states in the United States to gain a deeper understanding of the challenges for centers. For example, how do the centers address the needs of refugees? What do providers see as important components of culturally-effective services? What have they found to be successful approaches in bridging the cultural and linguistic challenges of providing services to an ever-changing refugee population?

\section{Method}

\section{Participants}

This purposive sample consisted of 15 mental-health workers in community health centers in all six New England states, including 12 social workers, 1 psychologist, 1 psychiatrist, and 1 program manager. These community centers are located in large and small cities where refugees are resettled in the largest numbers. The researchers contacted each center to invite the social worker - or, in the absence of a staff social worker, another professional in a similar role - to participate in the study. The sample consisted of 9 women, 6 men; three of the men had been refugees from countries in Southeast Asia or Africa. The participants worked in interdisciplinary teams with medical and behavioral health providers, and often held multiple roles: administrator, clinician, case manager, and program manager.

\section{Procedure}

The Institutional Review Board reviewed and approved the study. After having participants sign an informed consent, the researchers conducted one-time interviews held at each participant's health center. A semi-structured interview schedule was used, and the topics explored included: characteristics of the health center (e.g., services provided, funding sources), refugee populations served, training for practice with refugees, and approaches for addressing linguistic and cultural barriers. The interviews lasted between 60-90 minutes, and were digitally recorded and transcribed by a research assistant. 


\section{Data Analysis}

The interview transcriptions were uploaded to Dedoose, a qualitative data analysis application. Two research assistants were trained in the process of inductive qualitative data analysis from the Grounded Theory tradition (Bryant \& Charmaz, 2007). Both researchers and research assistants independently open-coded one interview; by comparing our respective coding decisions, we came to agreement on concepts that would be used by the research assistants to analyze the subsequent interviews. After coding the remaining transcripts, the researchers reviewed their data analysis, and together, we discussed their decisions and resolved any discrepancies. The research assistants were not involved in conducting the interviews, and thus, provided fresh perspectives on the data during the analysis. This team approach increased the trustworthiness and credibility of the analysis by allowing for greater fidelity in the coding decisions (Denzin \& Lincoln, 2011). In the next stage of analysis, the researchers expanded or collapsed concepts to ensure accuracy, and considered relationships across the concepts. Finally, exemplars were identified to reflect the concepts surfaced through the coding process.

\section{Findings}

In describing their work with refugee populations, three main themes arose across all interviews: client engagement as crucial; collaboration with interpreters; and cultural competence is an imperative but ill-defined. This last theme, cultural competence is an imperative but ill- defined, is an overarching theme that is woven throughout the other two. Client engagement as crucial seems to be a component of effective service delivery that the participants subjectively used as an assessment of their own cultural competency. Working with interpreters seemed to be a resource participants used as they tried to provide culturally competent services. The participants also acknowledged on-going challenges with being culturally competent, both on an individual level for the provider and on a systemic level throughout the center.

The participants identified the countries where their refugee populations originated from, including: Bhutan, Bosnia, Burundi, Burma, Cambodia, Congo, Djibouti, Eritrea, Iraq, Nepal, Somalia, and Sudan. New groups of refugees from new countries continue to arrive for services. Each community health center reported that the populations were everchanging, and most centers had no more than two weeks' notice of the new arrivals. This created a shared challenge among the participants as they felt ignorant of the historical and cultural experiences of the new arrivals, and ill-prepared to tailor services to them. Each theme will be described below with exemplars from the participants.

\section{Client Engagement as Crucial}

Participants reflected on the process of engagement with the client, and seemed to subjectively use their feelings of engagement with the client as an indicator of their own ability to bridge the cultural differences between themselves and the client. Participants understood that the journey for the refugees had been stressful. They also understood that many of the refugees felt mistrustful. The participants stated that engaging the client in

each session was a crucial component to effective interventions. Many health centers 
received a couple of weeks' notification that new refugees would arrive, rarely with any medical or behavioral health history. One participant explained: "We have a general sense in terms of numbers. Every few months we get an update from the [Office for Refugees and Immigrants] to get an idea of what to be ready for." This "general sense" seemed insufficient to fully anticipate and prepare for engagement with new refugee populations.

Client engagement often involved providing practical help to improve access and utilization of the health center's services. One participant explained, "The health center spends half a million dollars a year on what are called enabling services: interpretation and transportation." While resettlement agencies meet many of the refugees' concrete and immediate needs, the health centers tended to supplement these efforts with onsite assistance (e.g., a food pantry) and community referrals (e.g., English classes). A participant linked the provision of these services to their clients' clinical presentation and goals. At this center, social workers helped refugees obtain work experiences to help individuals feel more competent. "Whether volunteering or internships, it helps with mental health, gets people out of the house, helps get references, and maybe builds connections towards a job."

Participants spoke about the complexity of work, and thus, the need for collaboration with other providers. One participant described, "These are very time-consuming cases, and so if that team - everybody who is touching the case - doesn't have a forum to meet, then the case doesn't work." Another participant noted that social workers often are particularly skillful at client engagement, which can be modeled for a physician if they are both present. She explained the benefit of an integrated model that paired a clinician with a primary care team, "Very often the doctor will say, 'Oh, okay you go into room so and so.' Then that doctor doesn't see what that mental health person does. It's good for them to see what [the clinician] asks the person."

With effective client engagement, participants noticed that refugees knew they could turn to the health center for assistance. A participant described, "Our friends and colleagues in the Cambodian community said, 'There's a mental health crisis. Would you go over and take a look?" Refugees likely know the needs of the community more quickly than the clinicians. When a rapport has been established, refugees could partner with the center to meet their needs. For example, one participant described: "Most of our interpreters have approached us because they see the need that is in their own communities."

\section{Collaboration With Interpreters}

The theme of engagement was often interwoven with the participants' experiences with using interpreters in sessions with clients. Most of the participants were English-speaking and required interpreter services when working with clients. Health centers had different resources available for meeting this need, including bilingual staff who were trained as interpreters but also served other functions, part-time or per-diem professional medical interpreters, or telephone interpreters. Some centers had a combination of these options, depending on the needed language, availability of interpreters, and cost. A participant explained that his administrators favored phone interpretation for cost-savings: "Per-diem is expensive if there's a no-show or for just a few hours. They have to have a certain number 
of patients willing to be seen in order to justify a full-time interpreter."

Participants believed how the interpretation services were offered affected the quality of their interactions with the clients. This participant expressed the conflict between what the community health center could provide, versus what she believed enhanced client/provider engagement, "I get that the phone is cheaper, but I wish we could choose an interpretation service based on what is most helpful to establishing trust."

The participants varied in how they perceived the experience of in-person versus telephonic interpretation. For some, telephonic interpretation services were efficient and convenient, and allowed them to provide services for clients who spoke nearly any language. They also felt that it made the client feel safer than working with an in-person interpreter. Since many of the refugees fled due to internal conflicts, having someone from their culture and who lived in their current community, might feel unsafe, especially if the interpreter and client came from different sides of the conflict. One participant described, "We've found, most especially for our Iraqi patients, that they don't want an Arabicspeaking face-to-face interpreter. They prefer telephonic because that feels more protective and private." Another participant echoed this concern and described that patients sometimes insist keeping matters within their family unit:

Some of the cultures are a very close-knit group and they don't want this person to know their personal business, like our Nepali interpreter knows a lot of our Nepali patients, so they don't want him to know their business. They want their family member [to interpret].

For participants who favored telephone interpretation, they encouraged a greater engagement between the provider and client without an interpreter present. In these discussions, the participants returned to issues regarding engagement of the client, and how the use of an interpreter enhanced or impeded engagement. One participant reported that many of his colleagues felt interpreters were "in the way," and another wished that she could speak another language so as "not to have that third-party in the room." Others, however, experienced phone interpretation as a barrier to the therapeutic relationship because there can be difficulty hearing exactly what it being said, a lack of body language, and a lot of repetition. Another participant described the impact on client and provider engagement of using phone interpretation: "You sit there and you're really both looking at the phone, rather than interacting with each other. [The phone] is this plastic thing."

For the participants who preferred in-person interpretation, they believed the third person in the room enhanced engagement and made the visits go "smoother." Participants described several different roles that the interpreter played. Oftentimes, the interpreter shared cultural similarities to the client, and thus provided a sense of comfort and trust. In this sense, the interpreter "becomes part of the treatment team." All three partiesclinician, client, and interpreter - negotiate to find the most helpful balance between being a verbatim translator, a cultural broker, and a "clarifier when the terminology or jargon is too high." One participant described the process she engages in to determine if the arrangement is working: "It's always been a dialogue, and I always check in with the interpreter and the client to see what's going on, if I need more clarification or if they need clarification." Another participant echoed that it is helpful for the client and interpreter to 
"develop a rapport between them." Sometimes as the client feels more comfortable a shift can happen, and the client can engage with the clinician as well as the interpreter. A participant described:

Oftentimes because of the work we're doing, because of the trauma history, one of the shifts I'll notice is when the client starts to trust you. In the beginning they would just talk to the interpreter, and the interpreter talks back to me, but it's so cool to see that trust shifting, because then the person starts looking at me directly when they talk. It's that moment I know that the trust is building because it's awkward to talk to someone when they don't understand what you're saying, but that begins shift.

In this sense, the interpreter appears to meet needs of the client and the clinician, and assists with effective client engagement. Community health centers had difficulty finding qualified interpreters who also knew the community. Health center employees who spoke multiple languages were tapped to add interpretation work to their existing jobs on an adhoc basis, although this could be an imposition, may or may not be compensated work, or create a dual relationship. The participants were aware of the burden this can be on a staff worker, as this quote demonstrates,

Our front staff worker often is asked to interpret on-the-fly. It means that when she is called to interpret, either we have no one at the front desk, or we have to pull someone else in. And, you know, she doesn't get paid more for doing two jobs [interpreting and front desk]."

Another participant explained:

One of our case managers does [interpretation], but again, our case manager is very much in the community and has functions as a case manager here. We rarely use her as an interpreter for anything, because that complicates her life trying to do her actual job in terms of what she knows about their psychosocial needs, like food and housing.

\section{Cultural Competence is an Imperative but Ill-Defined}

While the participants reflected on the importance of engagement with the clients, and the challenges in using interpreters, these themes were parts of a larger theme: the need to be culturally competent yet with no clear definition or guidance on what constitutes cultural competence. The participants each expressed a need for cultural competence when working with refugees. In their assessments and interventions, participants found that some things were culturally unacceptable to inquire about, particularly mental health issues. A participant explained, "I did have one interpreter who said we don't ask about coping [coping is not a concept in the culture]. I tried to figure out another way to ask the same question." Another participant noted that the hesitancy goes beyond privacy concerns: "Sometimes people don't feel comfortable talking [about mental health], even if they don't know the interpreter." Participants also noted that health beliefs are culturally determined:

You could explain to them simple diabetes stuff, and diabetes is non-existent in some parts of Somalia. It's a challenge trying to convince them that there is 
treatment and medications for it, and people have lived with the disease 15, 20, 30 years, so [it seems like] nothing to worry about.

Cultural competence related to effective client engagement. Knowing the cultural norms for cross-gender encounters helped one female participant navigate seating arrangements:

I let the people choose whether they want to sit. If a male doesn't feel comfortable being in here with me alone, I often have a male interpreter. There is one man I keep the door half-open with because he doesn't feel comfortable being in here by himself.

The community health centers worked with refugees from several countries and experienced little training or information on the clients' needs, culture, or history. In addition, each client came to the center with multiple needs that reached beyond the services the centers could provide. This added stress to the participants who often felt ineffectual in bringing aboutchanges the refugees needed most to feel less anxious and depressed: housing or work.

Services would be improved if there were more trainings, education, and preparation for working with each group of refugees. While the participants assumed most of the refugees experienced some form of trauma prior to arriving, there were few other common characteristics among the different refugee populations. Participants stated that culture, language, and geographical differences made service delivery challenging as one approach was not universally effective. Knowing more about the specific group would enable the providers to know what questions to ask, what to look for in assessing trauma, and to better engage.

Participants saw themselves as responsible for becoming culturally competent in their work with refugee clients, especially as the countries of origin were regularly changing. One participant described learning from missteps early on. For example, she described, "When we got a new population, we were just looking around for somebody in the community who spoke Arabic, not realizing there's going to be all these different cultural issues." She learned from her clients and the interpreters in the community that the regional, ethnic, and religious differences needed to be considered in order to meet the clients' needs. One participant described that she could not tailor her work to a particular cultural group because they work with so many different, ever-changing groups. "I tend to use the same approach with everyone. I use cognitive behavioral therapy with my clients. I don't have the time to individualize, especially when we don't even speak the same language." While another participant believed that services needed to be targeted to each culture and felt limited by lack of time and training.

We're not familiar with any of our cultures. When I first came on board, I would spend an unbelievable amount of time before I met with a family researching that culture, but there's so many cultures that we work with that it's one of those things were I probably do less.

Some health centers had developed the cultural knowledge and skill set for working with a particular population, and while others had to constantly adapt to refugees from different countries of origin. 
Sometimes, demonstrating cultural competence meant changing aspects of how the center managed services. For example, one center set up blocks of time during the week when clients could drop-in as needed. This was initiated when refugees from some countries had a difficult time understanding the concept of "appointment time". Instead of having repeated no-shows from these clients, the clinic changed their concept of appointment. Providers aimed to engage refugee clients in a culturally effective manner, but at times encountered systemic issues that needed to be addressed for centers to be responsive and strategic with their limited funding.

\section{Discussion}

This study examined the views of 15 social service providers from 15 community health centers that serve refugees. The participants discussed challenges in providing culturally- effective care to a clientele that is ever-changing. The voices of the participants echo what many in community health centers and those who work with refugees know: the needs of this population are great, and the services few. Community health centers and other agencies that serve refugees and forced migrants are required to be culturally adept, flexible in the services provided and even in the structure of their agencies. These findings also highlight the need for more support to providers of refugee services.

There was a range of how each center described or demonstrated cultural competence. The range included, on one end, wall posters that presented ethnically diverse populations to, the other end, décor that reflected diverse cultures as well as bi-cultural staff, information posted in multiple languages, and in-person interpreters or bilingual staff. Some participants stated that they took extra measures to learn about the history of the refugee population. These participants wanted to know why the individual or family had to flee, which camps they were in while waiting for resettlement, and what their cultural beliefs were about health and well-being.

These differences in the participants' understanding of cultural competence was also evident in the interventions the participants used. For some participants, they felt that most of the refugees experienced depression and/or anxiety and would benefit from cognitive behavioral therapy (CBT). For these participants, CBT was effective across cultural differences. Those participants that believed culture differences affect the efficacy of an intervention, stated that they tried to understand the refugee's cultural beliefs about the presenting symptoms. These participants might use CBT and mindfulness practice, as well as draw from the refugee's own beliefs regarding healing and encourage the refugee to incorporate his/her own cultural remedies (i.e., coining, cupping).

\section{Limitations of the Study}

This study interviewed one primary provider in each of the 15 community health centers in this study. Interviewing more staff at each center might provide additional information and alternate voices than those captured in this study. This study did not interview interpreters or clients. Such interviews might offer important insights into how each member of the interventions (client, interpreter, social worker) experienced the quality and effectiveness of the services provided. The scope of this study was focused on the 
providers. Future studies should include recipients of refugee services. Studies that examined the satisfaction the recipients of the services experienced alongside the providers' perceptions of the effectiveness of the services might reveal interesting findings. In addition, the experiences and ability of multiple systems to provide culturally-effective services (providers, interpreters, governments, resettling agencies, communities) involved in providing for refugees should also be examined. Through such studies, a larger understanding of the challenges, opportunities, and current barriers to providing culturallyeffective services can be examined through a multi-systems and dynamic perspective that acknowledges the complexity of this global crisis

\section{Implications}

An important finding in this study is the acknowledged need by the participants that cultural competency is imperative to provide effective services, and the shared frustration among the respondents that that competency is difficult to define and harder to reach when working with multi-need clients from populations that are ever-changing. The participants from the 15 community health centers in this one region of the United States individually reported a similar challenge that service providers for refugees world-wide face: how to define cultural-competence and what do providers need to be competent. In this study, the participants focused on engagement as a critical component in cultural competency, and the use of interpreters as both a challenge and a conduit in developing engagement.

There are a number of on-going multi-system factors that complicate service provision to refugees. Community health centers and other agencies charged with providing services to refugees are often under-resourced and expected to be prepared with little to no advance notice or training. The global refugee crisis we face shows no signs of lessening. Agencies are asked to meet the needs of an ever-changing demographic without the resources, training, and combined applied knowledge that each center/agency requires. Current and future political conflicts and the increasing climate changes point toward a chronic refugee crisis. Social workers will continue to be called on to serve individuals and families who are forced to flee their homelands. It is imperative that social workers are prepared to meet this demand. Being prepared means better coordination of the involved systems (involved governments, resettlement agencies, communities), and embracing the practice of cultural competence within health and behavioral health teams. We do not need to acquire these skills in isolation from other agencies who provide for refugees. Sharing of experiences, such as in this study, can enhance our understanding of culturally-effective practice, and lessen the burden of learning new practices each time the population changes. It is hoped that this study widens the literature and that future studies will seek answers to questions such as: How does a profession learn to be culturally competent with as little as two-week notice of a new group's arrival? What are the most culturally effective ways of working with interpreters? What are the best approaches to engagement? Which interventions are most useful? The participants of this study offer us a window into these ongoing challenges, and deepen our understanding of cultural competence as an ethical imperative in addressing the needs of forced migrants. 


\section{References}

Adams, K. M., Gardiner, L. D., \& Assefi, N. (2004). Healthcare challenges from the developing world: Post-immigration refugee medicine. British Medical Journal, 328(7455), 1548-1552. doi: https://doi.org/10.1136/bmj.328.7455.1548

Al-Husban, M., \& Adams, C. (2016). Sustainable refugee migration: a rethink towards a positive capability approach. Sustainability, 8(5), 1-10. doi: https://doi.org/10.3390/su8050451

Al-Makhamreh, S., Spaneas, S., \& Neocleous, G. (2012). The need for political competence social work practice: Lessons learned from a collaborative project on Iraqi refugees-The case of Jordan. British Journal of Social Work, 42(6), 10741092. doi: https://doi.org/10.1093/bjsw/bcs087

Ay, M., Arcos González, P., \& Castro Delgado, R. (2016). The perceived barriers of access to health care among a group of non-camp Syrian refugees in Jordan. International Journal of Health Services, 46(3), 566-589. doi: https://doi.org/10.1177/0020731416636831

Barghadouch, A., Kristiansen, M., Jervelund, S. S., Hjern, A., Montgomery, E., \& Norredam, M. (2016). Refugee children have fewer contacts to psychiatric healthcare services: An analysis of a subset of refugee children compared to Danish-born peers. Social Psychiatry and Psychiatric Epidemiology, 51(8), 1125-1136. doi: https://doi.org/10.1007/s00127-016-1260-1

Bozorgmehr, K., Szecsenyi, J., Stock, C., \& Razum, O. (2016). Europe's response to the refugee crisis: Why relocation quotas will fail to achieve "fairness" from a health perspective. The European Journal of Public Health, 26(1), 5-6. doi: https://doi.org/10.1093/eurpub/ckv246

Bruno, A. (2015). Refugee admissions and resettlement policy. Current Politics and Economics of the United States, Canada and Mexico, 17(3), 485-501.

Bryant, A., \& Charmaz, K. (Eds.). (2007). The Sage handbook of grounded theory. Thousand Oaks, CA: Sage. doi: https://doi.org/10.4135/9781848607941

Burnett, A., \& Peel, M. (2001). Health needs of asylum seekers and refugees. British Medical Journal, 322, 544-547. doi: https://doi.org/10.1136/bmj.322.7285.544

Bustamante, L. H. U., Leclerc, E., Mari, J. D. J., \& Brietzke, E. (2016). It is time to prepare mental health services to attend to migrants and refugees. Revista Brasileira de Psiquiatria, 38(3), 263-264. doi: https://doi.org/10.1590/1516-4446-2015-1883

Campbell, J. R. (2014). Climate-change migration in the Pacific. The Contemporary Pacific, 26(1), 1-28. doi: https://doi.org/10.1353/cp.2014.0023

DeCola, A. (2011). Making language access to health care meaningful: The need for a federal health care interpreters' statute. Journal of Law \& Health, 24(1), 151182.

Denzin, N. K., \& Lincoln, Y. S. (Eds.). (2011). The Sage handbook of qualitative 
research. Thousand Oaks, CA: Sage.

Ellis, B. H., Murray, K., \& Barrett, C. (2014). Understanding the mental health of refugees: Trauma, stress, and the cultural context. In R. Parekh (Ed.), The Massachusetts General Hospital textbook on diversity and cultural sensitivity in mental health (pp. 165-187). New York: Springer. doi: https://doi.org/10.1007/978-1$\underline{4614-8918-4 \quad 7}$

Esses, V. M., Hamilton, L. K., \& Gaucher, D. (2017). The global refugee crisis: Empirical evidence and policy implications for improving public attitudes and facilitating refugee resettlement. Social Issues and Policy Review, 11(1), 78-123. doi: https://doi.org/10.1111/sipr.12028

Farbotko, C., Stratford, E., \& Lazrus, H. (2016). Climate migrants and new identities? The geopolitics of embracing or rejecting mobility. Social \& Cultural Geography, 17(4), 533-552. doi: https://doi.org/10.1080/14649365.2015.1089589

Fike, D. C., \& Androff, D. K. (2016). "The pain of exile": What social workers need to know about Burmese refugees. Social Work, 61(2), 127-135. doi: https://doi.org/10.1093/sw/sww005

Fondacaro, K. M., \& Harder, V. S. (2014). Connecting cultures: A training model promoting evidence-based psychological services for refugees. Training and Education in Professional Psychology, 8(4), 320-327. doi: https://doi.org/10.1037/tep0000071

Hansen, L., \& Huston, P. (2016). Health considerations in the Syrian refugee resettlement process in Canada. Canada Communicable Disease Report, 42(S2), S3-S7. doi: https://doi.org/10.14745/ccdr.v42is2a02

Healy, L. M. (2008). Exploring the history of social work as a human rights profession. International Social Work, 51(6), 735-748. doi: https://doi.org/10.1177/0020872808095247

Hsieh, E., \& Kramer, E. M. (2012). Medical interpreters as tools: Dangers and challenges in the utilitarian approach to interpreters' roles and functions. Patient education and counseling, 89(1), 158-162. doi: https://doi.org/10.1177/0020872808095247

Krogstad, J., \& Radford, J. (2017, January 30). Key facts about refugees to the U.S. Retrieved from http://www.pewresearch.org/fact-tank/2017/01/30/key-facts-aboutrefugees-to-the-u-s/

Matera, C., Stefanile, C., \& Brown, R. (2015). Majority-minority acculturation preferences concordance as an antecedent of attitudes towards immigrants: The mediating role of perceived symbolic threat and metastereotypes. International Journal of Intercultural Relations, 45, 96-103. doi: https://doi.org/10.1016/j.ijintrel.2015.02.001

Morland, L., \& Levine, T. (2016). Collaborating with refugee resettlement organizations: Providing a head start to young refugees. Young Children, 71(4), 69-75. 
Moulton, D. (2016). Refugee health clinics grapple with demand. Canadian Medical Association Journal, 188(11), E240-E240. doi: https://doi.org/10.1503/cmaj.109$\underline{5288}$

Njeru, J. W., DeJesus, R. S., Sauver, J. S., Rutten, L. J., Jacobson, D. J., Wilson, P., \& Wieland, M. L. (2016). Utilization of a mental health collaborative care model among patients who require interpreter services. International Journal of Mental Health Systems, 10(1), 15. 1-6. doi: https://doi.org/10.1186/s13033-016-0044-z

Sossou, M. A., Craig, C. D., Ogren, H., \& Schnak, M. (2008). A qualitative study of resilience factors of Bosnian refugee women resettled in the southern United States. Journal of Ethnic \& Cultural Diversity in Social Work, 17(4), 365-385. doi: https://doi.org/10.1080/15313200802467908

Taylor, J. (2004). The fundamentals of community health centers. Retrieved from https://www.nhpf.org/library/background-papers/BP_CHC_08-31-04.pdf

United Nations High Commissioner for Refugees. (2017). Figures at a glance. Retrieved from http://www.unhcr.org/en-us/figures-at-a-glance.html

Weaver, H. N. (2005). Reexamining what we think we know: A lesson learned from Tamil refugees. Affilia, 20(2), 238-245. doi: https://doi.org/10.1177/0886109905274546

Weine, S. M., Kuc, G., Dzudza, E., Razzano, L., \& Pavkovic, I. (2001). PTSD among Bosnian refugees: A survey of providers' knowledge, attitudes and service patterns. Community Mental Health Journal, 37(3), 261-271. doi: https://doi.org/10.1023/A:1017533214935

Yoosun, P. (2008). Making refugees: A historical discourse analysis of the construction of the 'refugee' in US social work, 1900-1957. British Journal of Social Work, 38(4), 771-787. doi: https://doi.org/10.1093/bjsw/ben015

Author note: Address correspondence to: Nicole Dubus, PhD, MSW, School of Social Work, San Jose State University, One Washington Square, San Jose, CA 95192, nicole.dubus@sjsu.edu 